\title{
杂多酸催化选择氧化甘油
}

韩布兴

中国科学院化学研究所, 胶体、界面与热力学实验室, 北京 100190

\section{Heteropolyacid Catalyzing the Selective Oxidation of Glycerol}

\section{HAN Buxing}

CAS Key Laboratory of Colloid and Interface Thermodynamics, Institute of Chemistry, Chinese Academy of Sciences, Beijing 100190, P. R. China.

E-mail: hanbx@iccas.ac.cn

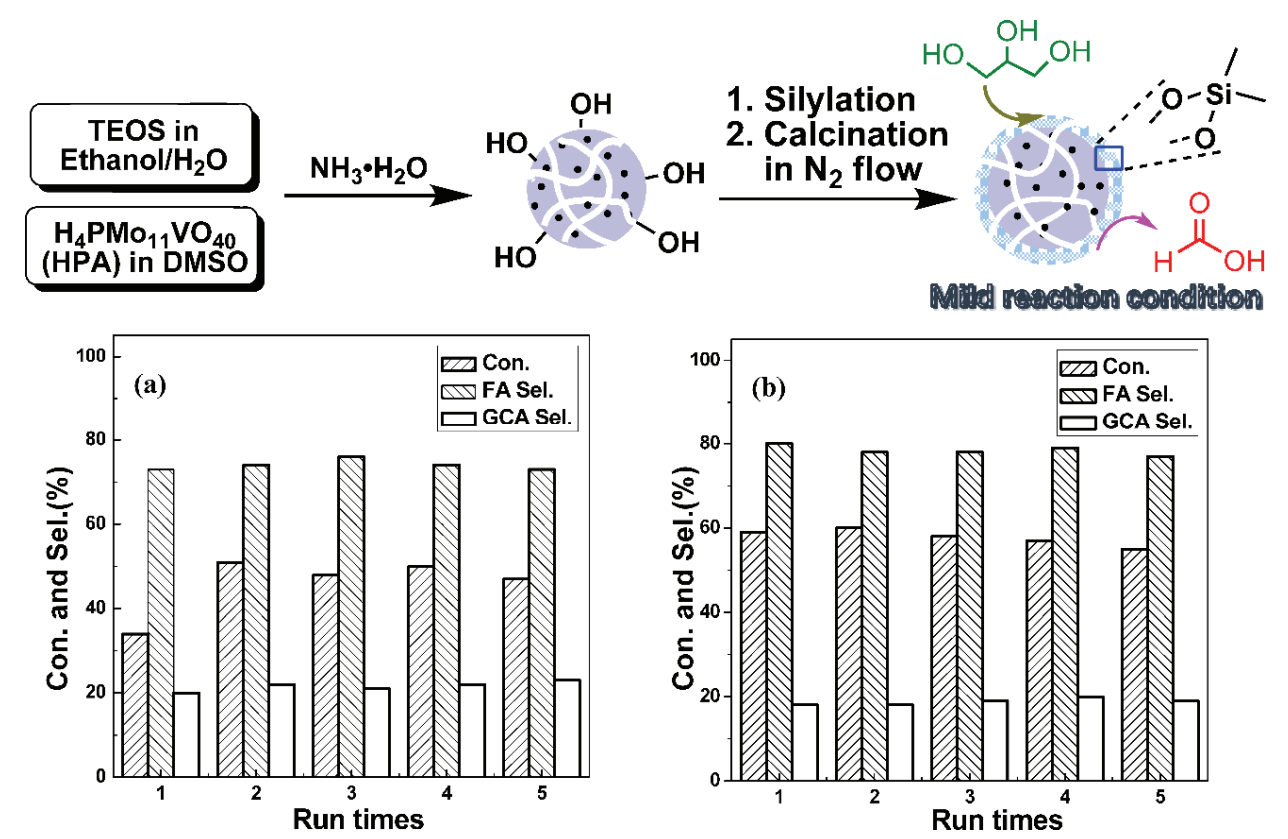

上图: 二氧化硅包覆杂多酸催化剂的制备途径; 下图: 催化剂的循环利用性, 无酸添加(a)和添加三氟甲磺酸(b)。

近几十年来, 生物柴油作为绿色的生物燃料 的技术日趋成熟, 在整个生物柴油生产过程中, 会 产生大约 $10 \%(w$, 质量分数)的副产物甘油, 因此 通过催化选择氧化甘油过程, 获得高附加值化学 品引起人们极大的关注。研究表明负载型贵金属 催化剂对甘油氧化过程呈现出较高活性和选择 性 ${ }^{1,2}$, 但是反应通常需要在碱性条件下进行, 因此 反应后会产生大量的有机酸盐, 反应混合物需要 进一步中和酸化, 才能得到有机酸的目标产物。另 外, 贵金属催化剂表面受到过度氧化后可能失 活 $^{3}$, 而且负载型贵金属催化剂在实际应用中价格
昂贵。因此, 设计更高效、低成本的选择氧化催化 剂体系成为当前研究领域的一个热点。除了分子 氧之外, 过氧化氢作为一种强氧化剂, 在较温和的 反应条件下被广泛应用于各类有机氧化反应中, 它的降解产物只有氧气和水 ${ }^{4}$, 因此, 近年来人们 对过氧化氢作为氧化剂对甘油进行选择性氧化的 路径也展开了探索 ${ }^{5-7}$ 。

杂多酸是一类具有强酸性和优良的氧化还原 性的化合物, 具有容易得到、结构稳定的优点, 因 而杂多酸在催化生物质分子氧化转化领域备受关 注 $^{8}$ 。虽然杂多酸作为一种高效催化剂在甘油水溶 
液中催化甘油生成甲酸的研究已有报道 ${ }^{9}$, 但是杂 多酸在水溶液中具有很高的溶解度, 所以催化剂 的清洁高效分离是一个亟待解决的问题。为了解 决杂多酸催化剂的循环利用问题, 华东理工大学 侯震山教授课题组成员利用溶胶-凝胶法和硅烷 化方法将杂多酸催化剂封装在二氧化硅载体内 部, 随后以傅立叶红外光谱、X射线衍射仪、热重 分析仪、透射电子显微镜、扫描电镜等手段对合成 的材料进行了表征。红外光谱表明杂多酸在催化 剂中保持了其完整的Keggin结构, X射线衍射、透 射电镜表征证明杂多酸高度分散在二氧化硅载体 中, 扫描电镜表征显示催化剂呈球形纳米颗粒形 貌。他们随后将二氧化硅封装的杂多酸催化剂应 用于甘油氧化反应中, 以过氧化氢为氧化剂, 在温 和的反应条件下, 合成的材料对甘油氧化具有良 好的催化活性, 其中对甲酸的选择性大约为 $70 \%$, 对乙醇酸的选择性大约为 $27 \%$, 反应之后的催化剂 不仅可以回收, 而且催化剂经过一次循环后, 转化 率由第一次的 $34 \%$ 提高到 $50 \%$ 左右, 对产物的选择 性基本保持不变。侯震山教授课题组成员发现硅 烷化的后处理过程对于催化剂循环起着重要的作 用, 单纯二氧化硅的比表面积为 $287 \mathrm{~m}^{2} \cdot \mathrm{g}^{-1}$, 二氧 化硅包覆杂多酸经过硅烷化后, 其比表面积降为 $245 \mathrm{~m}^{2} \cdot \mathrm{g}^{-1}$, 而且孔径也有所降低。不仅如此, 他 们还发现单纯二氧化硅与水的接触角为 $0^{\circ}$, 而二 氧化硅包覆的杂多酸在硅烷化之后的催化剂具有 很强的疏水性, 与水的接触角达到 $137^{\circ}$ 。这些表征 结果说明硅烷化过程不仅可以显著提高催化剂的 疏水性, 而且同时缩小了载体孔径, 可阻止杂多酸 流失到反应体系中, 从而使杂多酸被限制在二氧 化硅载体中, 实现催化剂的回收利用。为了揭示酸 在甘油氧化过程中对甘油分子的作用, 在反应体 系中加入 Lewis 或者Brönsted 酸, 发现加入强 Brönsted酸可以显著提高反应的催化性能, 从而说 明质子酸对甘油分子的活化起着重要的作用。 该工作已在物理化学学报上在线发表(doi: 10.3866/PKU.WHXB201711151), 为特刊 “绿色化 学” 邀请的原创文章 ${ }^{10}$ 。该工作进一步拓展了杂多 酸催化剂催化选择氧化甘油生成甲酸的研究, 该 体系最大优势在于甘油选择氧化反应可以在非常 温和条件下进行, 且催化剂能够实现循环利用。

\section{References}

(1) Villa, A.; Dimitratos, N.; Chan-Thaw, C. E.; Hammond, C.; Prati, L.; Hutchings, G. J. Acc. Chem. Res. 2015, 48 (5), 1403. doi: $10.1021 / \mathrm{ar} 500426 \mathrm{~g}$

(2) Zhou, C. H.; Zhao, H.; Tong, D. S.; Wu, L. M.; Yu, W. H. Catal. Rev. Sci. Eng. 2013, 55 (4), 369. doi: 10.1080/01614940.2013.816610

(3) Davis, S. E.; Ide, M. S.; Davis, R. J. Green Chem. 2013, 15 (1), 17. doi: $10.1039 / \mathrm{c} 2 \mathrm{gc} 36441 \mathrm{~g}$

(4) Campos-Martin, J. M.; Blanco-Brieva, G.; Fierro, J. L. Angew. Chem. Int. Ed. 2006, 45 (42), 6962. doi: 10.1002/anie.200503779

(5) Sarkar, B.; Pendem, C.; Konathala, L. N. S.; Tiwari, R.; Sasaki, T.; Bal, R. Chem. Commun. 2014, 50 (68), 9707. doi: $10.1039 / \mathrm{c} 4 \mathrm{cc} 03842 \mathrm{~h}$

(6) Faroppa, M. L.; Musci, J. J.; Chiosso, M. E.; Caggiano, C. G.; Bideberripe, H. P.; Fierro, J. L. G.; Siri, G. J.; Casella, M. L. Chin. J. Catal. 2016, 37 (11), 1982. doi: 10.1016/S1872-2067(16)62531-7

(7) Corrado Crotti, C.; Farnetti, E. J. Mol. Catal. A-Chem. 2015, 396, 353. doi: 10.1016/j.molcata.2014.10.021

(8) Lu, T.; Niu, M.; Hou, Y.; Wu, W.; Ren, S.; Yang, F. Green Chem. 2016, 18 (17), 4725. doi: 10.1039/c6gc01271j

(9) Lan, J.; Lin, J.; Chen, Z.; Yin, G. ACS Catal. 2015, 5 (4), 2035. doi: $10.1021 / \operatorname{cs} 501776 \mathrm{n}$

(10) Yuan, M.; Li, D.; Zhao, X.; Ma, W.; Kong, K.; Ni, W.; Gu, Q.; Hou, Z. Acta Phys. -Chim. Sin. 2018, 34 (8), 886. [袁明明, 李迪帆, 赵秀 阁, 马文保, 孔康, 倪文秀, 顾青雯, 侯震山. 物理化学学报, 2018, 34 (8), 886.] doi: 10.3866/PKU.WHXB201711151 